\title{
Perfumes of survival
}

\author{
Peter Nick ${ }^{1}$
}

(C) Springer-Verlag Wien 2015

Plants are smart. They were able to circumvent the limitations of their sessile lifestyle by mainly two inventions: a robust development that can adapt to a broad range of environmental conditions and a sophisticated manipulation of other (more mobile) organisms that will then volunteer to serve the needs of the immobile plants. These services are often compensated by a reward, creating a compromise that is favourable for both partners and therefore very sustainable in most cases. The coevolution of flowering plants and their pollinators provides the best-known example of this co-evolutionary success story. Driven by technological advances in chemical analytics, over the last decade, numerous and sometimes breathtaking facets of these supra-organismic interactions have been discovered. Unlike animals, plants cannot use movements as carrier for signals. Moreover, their responses fall into a different time scale (hours or days rather than milliseconds or seconds). Behaviour, the central means of animal communication, is therefore replaced by chemical signals. The need to rely on manifold and specific chemical signals to manipulate the interaction with other organisms, has, presumably, been one of the major forces driving the evolution of the proficient secondary metabolism characteristic for plants.

While we have learnt a lot about plants as masters of secret chemical manipulation, this knowledge creates novel questions. A central question is how such a degree of sophistication has been able to evolve - it is extremely unlikely that this happened in a single event, but if it happened over several steps, how were these intermediate situations stabilised then?

\section{Peter Nick}

peter.nick@kit.edu

1 Molecular Cell Biology, Botanical Institute, Karlsruhe Institute of Technology, Karlsruhe, Germany
A precursor of a signal that is not acting as signal itself should have disappeared because it would not confer a selective advantage. The fact that it might confer a selective advantage some day in a future generation, after being extended by the subsequent step (still to be developed in the future), cannot explain how and why this trait should be stable now and here. The concept of preadaptation allows to escape this conceptual dilemma: a trait seldom confers only a single function, and while it might be positively selected for one of these functions during an early stage of evolution, a second function, originally playing a minor role, can shift into the focus of selection, when the organism is facing a new environment. Such functional shifts are especially frequent for those enzymes that underlie secondary metabolism, since most of these enzymes accept several substrates, often with only vague preferences for the main substrate. If, by a small change in the flow of metabolic precursors, a novel or so far rare substrate becomes abundant, very often entire assemblies of products can arise, a phenomenon that has often been termed as sleeping metabolic potential.

Molecules that enter the gas phase at ambient temperatures are most efficient as inter-organismic signals, because they can bridge large distances and also dissipate rapidly, as soon as their synthesis ceases (to switch off a signal is probably at least as important as to activate it). Two contributions in the current issue add new aspects into the fascinating world of plant volatiles and also bear promising potential for biotechnological application:

The large number of compounds that have evolved to target defined interaction partners can be perceived as something like a natural chemical library where specificity has been a priority during its construction. Using this strategy, Sunohara et al. 2015 in the current issue were screening medical plants for volatile compounds that act as so-called allelopathic compounds and suppress germination and development of competing plant species. These phytotoxins have therefore some 
potential to be developed into specific and environmentally safe herbicides. In fact, the screen of 71 medical plant species led to the identification of several monoterpenes with phytotoxic activity. Some of these compounds, such as $\beta$-pinene, had already been known to harbour allelopathic activity (Chowhan et al. 2013), but some were novel. Among these novel compounds, eucarvone turned out to be very promising, because it showed a pronounced species dependence of its effect. Whereas in sensitive target species, such as the pertinent weed finger-millet, eucarvone was activating an oxidative burst followed by cell death in seedling roots, the crop plant maize did not show these symptoms, rendering this compound into a novel lead structure for the development of a new generation of environmentally friendly and specific herbicides.

The work by Lee et al. 2015 in the current issue investigates the volatiles produced by the important crop plant rice and uncovers interesting insights into a potential preadaptation scenario explaining, how these compounds have turned into signals for biotic interaction. Again, the dominant volatiles belong to the monoterpenes. These derivatives of the isoprenoid pathway are generated from a side branch of carotenoid synthesis. Carotenoids are evolutionary ancient, and since they are endowed with numerous conjugated double bonds, they are ideal scavengers of reactive oxygen species, a hazardous by-product of photosynthesis under strong light. When subjecting rice seedlings to ionising radiation (UV-B or $\gamma$-rays), but also when imposing oxidative stress using hydrogen peroxide, authors observe identical bouquets of monoterpenes with $(S)$-limonene as lead compound. Using reverse genetics, they then isolate a rice terpene synthase and show after recombinant expression that this protein in fact converts the substrate geranyl diphosphate into $(S)$-limonene. They follow then the expression of this gene and can demonstrate that its transcripts accumulate under the same conditions where the enzymatic product, $(S)$-limonene, is formed. They interpret their findings in terms of a function of these volatiles as antioxidants that can mitigate stressors linked with strong solar irradiation such as ozone, photosynthetic disbalance, or heat. This explanation bears interesting conceptual consequences - have these powerful chemical manipulators originally evolved by branching off the ancient photooxidative protection system? Their volatile nature would have allowed for rapid spread within the tissue and therefore global protection of the stressed leaves. In the moment, where such a volatile evoked a response in a different organism, and where this response was beneficial for the producing plant, this would channel a functional shift, where the "hitchhiking" novel function (modulation of a biotic interaction) would have been positively selected and eventually turn into the main function of these terpenoids.

Conflict of interest The author declares that he has no competing interests.

\section{References}

Chowhan N, Singh HP, Batish DR, Kaur S, Ahuja N, Kohli RK (2013) $\beta$ Pinene inhibited germination and early growth involves membrane peroxidation. Protoplasma 250:691-700

Lee GW, Lee SB, Chung MS, Jeong YS, Chung BY (2015) Rice terpene synthase 20 (OsTPS20) plays an important role in producing terpene volatiles in response to abiotic stresses. Protoplasma, current issue

Sunohara Y, Baba Y, Matsuyama S, Fujimura K, Matsumoto H (2015) Screening and identification of phytotoxic volatile compounds in medicinal plants, and characterizations of a selected compound, eucarvone. Protoplasma, current issue 